\title{
Equivalence of Nyström's Method and Fourier Methods for the Numerical Solution of Fredholm Integral Equations
}

\author{
By Jean-Paul Berrut* and Manfred R. Trummer**
}

\begin{abstract}
Nyström's method with the trapezoidal rule, and the Fourier method, produce the same approximation to the solution of an integral equation at the collocation points for Nyström's method. This equivalence allows the derivation of error estimates for Nyström's method, and gives an intuitive explanation for its good performance in the periodic case. The equivalence holds for Fourier methods with arbitrary orthogonal basis functions. The quadrature rule for numerical integration must have the collocation points as abscissae, and must yield the exact entries of the Gramian matrix of the orthogonal basis.
\end{abstract}

1. Description of the Fourier Method. In this note we will prove a result on the equivalence of Nyström's method and the Fourier method with orthogonal functions for the numerical solution of Fredholm integral equations. We encountered this question in the course of implementing the conformal mapping method described in [10], [16] with Fourier methods; see also [3], [4].

We will assume throughout that the discretized versions of integral equation (2) below have a unique solution. The restriction to the interval $[0,2 \pi]$ is only for the sake of convenience. Let $h \in L^{2}([0,2 \pi] \times[0,2 \pi])$ be a Hilbert-Schmidt kernel defining the Hilbert-Schmidt integral operator

$$
\mathbf{H}: f \in L^{2} \rightarrow \mathbf{H} f:=\frac{1}{2 \pi} \int_{0}^{2 \pi} h(\cdot, s) f(s) d s \in L^{2} .
$$

Let $g \in L^{2}[0,2 \pi]$, and consider the Fredholm integral equation in $L^{2}[0,2 \pi]$,

$$
\lambda f+\mathbf{H} f=g,
$$

for an unknown $f \in L^{2}[0,2 \pi] . \lambda$ is a scalar, real or complex.

If $h$ and $g$ are $2 \pi$-periodic, an appealing procedure for solving (2) numerically is the Fourier method, which can be described as follows. We choose $N$ equidistant

Received December 5, 1984; revised May 19, 1986.

1980 Mathematics Subject Classification. Primary 65R20, 45L10, 42A10.

Key words and phrases. Integral equations, Nyström's method, Fourier method, collocation, trigonometric approximation.

*This work was performed at the Seminar für Angewandte Mathematik, ETH Zürich, Switzerland.

**Research partially supported by a postdoctoral fellowship under NSERC (Canada) grant A8240. 
interpolating points $s_{j}:=t_{j}:=2 \pi j / N$, and interpolate the kernel $h$ and the inhomogeneity $g$ by trigonometric polynomials***

$$
\begin{gathered}
\hat{h}(t, s):=\sum_{n=-M^{\prime}}^{M} \sum_{m=-M}^{M^{\prime}} a_{n m} e^{i n t} e^{i m s}, \\
\hat{g}(t):=\sum_{n=-M^{\prime}}^{M} d_{n} e^{i n t},
\end{gathered}
$$

where

$$
M^{\prime}:= \begin{cases}M, & \text { if } N \text { is odd }, N=2 M+1 \\ M-1, & \text { if } N \text { is even, } N=2 M\end{cases}
$$

The coefficients $a_{n m}$ and $d_{n}$ are the elements of the discrete Fourier transforms (DFTs) [7]

$$
\begin{aligned}
& \mathbf{a}:=F_{N}^{(2)} \mathbf{h} \in \Pi_{N}^{(2)} \quad \text { (two-dimensional), } \\
& \mathbf{d}:=F_{N} \mathbf{g} \in \Pi_{N} \quad \text { (one-dimensional), }
\end{aligned}
$$

of the bi-infinite $N$-periodic sequences $\mathbf{h} \in \Pi_{N}^{(2)}$ and $\mathbf{g} \in \Pi_{N}$ of the interpolated values $h_{j k}:=h\left(t_{j}, s_{k}\right)$ and $g_{j}:=g\left(t_{j}\right)[7$, p. 495],

$$
\begin{gathered}
a_{n m}=\frac{1}{N^{2}} \sum_{j, k=0}^{N-1} h_{j k} w^{-j n} w^{-k m} \\
d_{n}=\frac{1}{N} \sum_{j=0}^{N-1} g_{j} w^{-j n}
\end{gathered}
$$

where $w:=\exp (2 \pi i / N)$. We seek a solution of the form

$$
\hat{f}(t):=\sum_{n=-M^{\prime}}^{M} b_{n} e^{i n t}
$$

with unknown coefficients b. The values of $\hat{f}$ at the collocation points, $\hat{f}_{j}=\hat{f}\left(t_{j}\right)$, can be expressed as the inverse DFT of the coefficients $b_{n}, \hat{\mathbf{f}}=F_{N}^{[-1]} \mathbf{b}$. Replacing the functions $f, g$, and $h$ in (2) by their approximations $\hat{f}, \hat{g}$, and $\hat{h}$, and taking into account the orthogonality of the basis functions $e^{i n t}$, we obtain the following system of linear equations for the $b_{n}$ :

$$
\lambda b_{n}+\sum_{m=-M}^{M^{\prime}} a_{n m} b_{-m}=d_{n}, \quad n=-M^{\prime}, \ldots, M .
$$

In Section 3 we shall extend the above method to orthonormal Chebyshev systems, and show its equivalence to Nyström's method. However, this special case is important enough to deserve special attention. Moreover, the powerful tools of Fourier analysis allow a quite elegant proof of the equivalence via the convolution theorem.

\footnotetext{
*** Our notation suppresses the dependence of $\hat{h}$ and $\hat{g}$ on $N$.
} 
2. Equivalence of the Nyström and the Fourier Method. The linear system (7) arising from the Fourier method is mathematically equivalent to the system obtained by Nyström's method. Using the definition (5a) of the Fourier coefficients we get

$$
\begin{aligned}
\sum_{m=-M}^{M^{\prime}} a_{n m} b_{-m} & =\sum_{m=-M}^{\prime} \frac{1}{N^{2}} \sum_{j, k=0}^{N-1} h_{j k} w^{-j n} w^{-k m} b_{-m} \\
& =\frac{1}{N} \sum_{j=0}^{N-1}\left[\sum_{m=-M}^{M^{\prime}} \frac{1}{N} \sum_{k=0}^{N-1} h_{j k} w^{-k m} b_{-m}\right] w^{-j n} \\
& =\frac{1}{N} \sum_{j=0}^{N-1}\left[\sum_{m=-M}^{M^{\prime}} r_{m}^{(j)} b_{-m}\right] w^{-j n} .
\end{aligned}
$$

The $r_{m}^{(j)}$ are the elements of the one-dimensional DFT of the $j$ th "row" $\mathbf{h}^{(j)}:=\left\{h_{j k}\right\}_{k=-\infty}^{\infty}$ of $\mathbf{h}=\left\{h_{j k}\right\}_{j, k=-\infty}^{\infty}$,

$$
\mathbf{r}^{(j)}:=F_{N} \mathbf{h}^{(j)} .
$$

In other words, the $r_{m}^{(j)}$ are the coefficients of the trigonometric polynomial which interpolates the univariate function $s \rightarrow h\left(t_{j}, s\right)$ at the points $s_{k}$. We remark that (8) leads to a very efficient implementation of the Fourier method, avoiding two-dimensional DFTs [3].

In view of the $N$-periodicity of the sequences involved, the last expression in brackets in (8) equals

$$
\sum_{m=0}^{N-1} r_{m}^{(j)} b_{-m}
$$

which is the zeroth element of the convolution [7, p. 508] of the DFTs of the two sequences $\mathbf{h}^{(j)}$ and $\hat{\mathbf{f}}$. The convolution theorem then yields

$$
\begin{aligned}
\sum_{m=-M}^{M^{\prime}} r_{m}^{(j)} b_{-m} & =\left(F_{N} \mathbf{h}^{(j)} * F_{N} \hat{\mathbf{f}}\right)_{0}=\left(F_{N}\left(\mathbf{h}^{(j)} \cdot \hat{\mathbf{f}}\right)\right)_{0} \\
& =\frac{1}{N} \sum_{k=0}^{N-1} h\left(t_{j}, s_{k}\right) \hat{f}_{k},
\end{aligned}
$$

where $\mathbf{h}^{(j)} \cdot \hat{\mathbf{f}}$ denotes the Hadamard product of $\mathbf{h}^{(j)}$ and $\hat{\mathbf{f}}$. According to (8), the matrix-vector product in Eq. (7) is the DFT of the sequence whose elements are the right-hand side of (9) for $j=0, \ldots, N-1$. Thus, by applying the inverse DFT, $F_{N}^{[-1]}$, to both sides of (7), we finally obtain

$$
\lambda \hat{f}_{j}+\frac{1}{N} \sum_{k=0}^{N-1} h\left(t_{j}, s_{k}\right) \hat{f}_{k}=g_{j}, \quad j=0, \ldots, N-1 .
$$

This is precisely the system of equations of Nyström's method [2] with equidistant collocation points and the trapezoidal rule as quadrature formula. Thus, we have proved

THEOREM 1. The values of the solution at the collocation (interpolation) points obtained by Nyström's method with the trapezoidal rule are identical to the values obtained by the Fourier method. 
Before we proceed to the next section, some comments on the above equivalence are in order.

(a) This equivalence provides an intuitive explanation why one cannot expect good results from Nyström's method with the trapezoidal rule unless the kernel and the inhomogeneity of the integral equation are periodic. On the other hand, in the periodic case, Nyström's method is preferable with respect to computing time, because no DFTs have to be computed. This saves $O\left(N^{2} \log N\right)$ operations. The values of the interpolating trigonometric polynomial at intermediate points $t \neq t_{k}$ can then be computed very efficiently by barycentric formulas [9], [5].

(b) The equivalence result does not make the Fourier method obsolete. There are situations where Nyström's method is not applicable, but the Fourier method is. One example is the case of weakly singular equations, which play quite a prominent role in numerical conformal mapping and two-dimensional boundary value problems [15], [12], [3]. Another example is provided in [4], where the kernel is not given explicitly, but defined as the harmonic conjugate of a known function.

(c) The equivalence is also interesting from a theoretical point of view. It allows a simple proof of the exponential asymptotic convergence of Nyström's method if both the kernel and the inhomogeneity are analytic. Indeed, if the operator $\mathbf{I}+\mathbf{H}$ is bijective in $L^{2}[0,2 \pi]$, and if $\|\hat{h}-h\|_{2} \rightarrow 0,\|\hat{g}-g\|_{2} \rightarrow 0$, then, for $N$ sufficiently large, (7) has a unique solution $\hat{f}$, and the $\hat{f}$ converge to the exact solution $f$ in the $L^{2}$-norm. Moreover, the estimate

$$
\|\hat{f}-f\|_{2} \leqslant C\left(\|\hat{h}-h\|_{2}+\|\hat{g}-g\|_{2}\right)
$$

holds with a constant $C$ depending only on $\left\|(\mathbf{I}+\mathbf{H})^{-1}\right\|_{2}$ and $\|\hat{g}\|_{2}$ (cf. [13, p. 480]). If $h$ and $g$ are periodic and analytic, then the convergence of $\hat{h}$ and $\hat{g}$ to $h$ and $g$, respectively, is exponentially fast (cf. the lemma on p. 490 in [7]).

(d) If $N$ is even, the interpolating polynomials $\hat{h}$ and $\hat{g}$ of degree $M$ are not unique; instead of (3), one usually chooses the so-called balanced trigonometric polynomials (cf. [8]). However, except in extreme cases where the exact coefficients with index $\pm M$ are dominant, the difference between the two approaches is negligible. Indeed, in view of the aliasing formula [7, p. 489], the error committed in replacing a function by its interpolating trigonometric polynomial is of the order of magnitude of the computed coefficients with greatest indices [3].

3. Generalization to Orthonormal Chebyshev Systems. In the sequel we will refer to Fourier type methods as the Fourier method described above with the exponentials replaced by any orthonormal basis $\phi_{1}, \ldots, \phi_{N}$ in $L^{2}[0,2 \pi]$. The Fourier method can be viewed as a discrete Galerkin method with the $\phi_{j}$ serving as test and trial functions. However, in the Fourier method the kernel and the right-hand side are approximated by a linear combination of the $\phi_{j}$, and all subsequent calculations are performed in Fourier space. Thus, the Fourier method differs from the standard Galerkin method as described in [2], [6]. The $N$ collocation points $t_{j}{ }^{\dagger}$ and the $N$ weights $w_{j}, 1 \leqslant j \leqslant N$, define the quadrature rule $Q$ by

$$
Q \psi:=\sum_{j=1}^{N} w_{j} \psi\left(t_{j}\right) \approx \frac{1}{2 \pi} \int_{0}^{2 \pi} \psi(s) d s .
$$

\footnotetext{
${ }^{\dagger}$ The $t_{j}$ are no longer assumed to be equidistant.
} 
These collocation points and the quadrature rule $Q$ are used in Nyström's method, leading to the system of linear equations

$$
\lambda x_{i}+\sum_{j=1}^{N} w_{j} h\left(t_{i}, t_{j}\right) x_{j}=g\left(t_{i}\right), \quad 1 \leqslant i \leqslant N,
$$

where the $x_{i}$ are approximations to $f\left(t_{i}\right)$.

On the other hand, $Q$ is also used to compute the "Fourier coefficients" with respect to our orthonormal basis $\phi_{1}, \ldots, \phi_{N}$. The space $L^{2}[0,2 \pi]$ is endowed with the scalar product

$$
\langle f, g\rangle:=\frac{1}{2 \pi} \int_{0}^{2 \pi} f(t) \overline{g(t)} d t .
$$

Let $\phi_{1}, \ldots, \phi_{N}$ be an orthonormal Chebyshev system (see, e.g., [14]). We attempt to approximate the solution in the subspace $\Phi_{N}$ spanned by the $\phi_{i}$ :

$$
f(t) \approx \sum_{j=1}^{N} b_{j} \phi_{j}(t)
$$

To that end, we approximate $g$ and $h$ by the functions $\hat{g}$ and $\hat{h}$ in $\Phi_{N}$ and $\Phi_{N} \times \Phi_{N}$, respectively,

$$
\hat{g}(t)=\sum_{i=1}^{N} d_{i} \phi_{i}(t), \quad \hat{h}(t, s)=\sum_{i=1}^{N} \sum_{j=1}^{N} a_{i j} \phi_{i}(t) \phi_{j}(s) .
$$

Taking $\left\langle g, \phi_{i}\right\rangle$ for the $d_{i}$ and $\left\langle\phi_{i}, \mathbf{H} \phi_{j}\right\rangle$ for the $a_{i j}$ gives the best approximations in $\Phi_{N}$ in the $L^{2}$ sense. However, by using our quadrature formula (12) to evaluate these scalar products, we obtain

$$
a_{i j}=\sum_{p=1}^{N} \sum_{q=1}^{N} w_{p} \overline{\phi_{i}\left(t_{p}\right)} h\left(t_{p}, t_{q}\right) \overline{\phi_{j}\left(t_{q}\right)} w_{q}
$$

and

$$
d_{i}=\sum_{p=1}^{N} w_{p} \overline{\phi_{i}\left(t_{p}\right)} g\left(t_{p}\right)
$$

Equation (2) is then approximated by the linear system of equations (cf. (7))

$$
(\lambda I+A) \mathbf{b}=\mathbf{d} .
$$

THEOREM 2. Let $\left\{\phi_{i}\right\}, 1 \leqslant i \leqslant N$, be an orthonormal Chebyshev system, and suppose the quadrature rule (12) has nonzero weights and evaluates the Gramian matrix $\left\langle\phi_{i}, \phi_{j}\right\rangle$ exactly, i.e.,

$$
\sum_{p=1}^{N} w_{p} \phi_{i}\left(t_{p}\right) \overline{\phi_{j}\left(t_{p}\right)}=\delta_{i j}
$$

where $\delta_{i j}$ is the Kronecker symbol. Then the values of the solution at the points $t_{j}$ obtained by the Fourier method described above are identical to the values $x_{j}$ obtained by Nyström's method.

Proof. We start by showing that the Fourier method is actually equivalent to the standard Galerkin scheme under the conditions stated. The key observation is, that the numerical approximations to $\left\langle g-\hat{g}, \phi_{i}\right\rangle$ and $\left\langle(\mathbf{H}-\hat{\mathbf{H}}) \phi_{i}, \phi_{j}\right\rangle$ vanish because of the orthogonality relation (19). $\hat{\mathbf{H}}$ denotes the integral operator with kernel $\hat{h}$. We 
write down the explicit formula only for the first scalar product:

$$
\begin{aligned}
\sum_{q=1}^{N} w_{q} \overline{\phi_{j}\left(t_{q}\right)} \hat{g}\left(t_{q}\right) & =\sum_{q=1}^{N} w_{q} \overline{\phi_{j}\left(t_{q}\right)}\left(\sum_{i=1}^{N} d_{i} \phi_{i}\left(t_{q}\right)\right) \\
& =\sum_{q=1}^{N} w_{q} \overline{\phi_{j}\left(t_{q}\right)}\left(\sum_{i=1}^{N} \sum_{p=1}^{N} w_{p} \overline{\phi_{i}\left(t_{p}\right)} g\left(t_{p}\right) \phi_{i}\left(t_{q}\right)\right) \\
& =\sum_{i=1}^{N} \sum_{p=1}^{N}\left(\sum_{q=1}^{N} w_{q} \overline{\phi_{j}\left(t_{q}\right)} \phi_{i}\left(t_{q}\right)\right) w_{p} \overline{\phi_{i}\left(t_{p}\right)} g\left(t_{p}\right) \\
& =\sum_{p=1}^{N} w_{p} \overline{\phi_{j}\left(t_{p}\right)} g\left(t_{p}\right)
\end{aligned}
$$

because the inner sum in the last equation equals $\delta_{i j}$. Therefore (18) is equivalent to the system of equations arising from the standard (discrete) Galerkin scheme

$$
\sum_{p=1}^{N} w_{p}\left(\lambda x_{p}+\sum_{q=1}^{N} w_{q} h\left(t_{p}, t_{q}\right) x_{q}-g\left(t_{p}\right)\right) \overline{\phi_{j}\left(t_{p}\right)}=0
$$

where the $x_{i}$ are again approximations to $f\left(t_{i}\right)$. Obviously, if $x_{i}, i=1, \ldots, N$, is a solution of the Nyström system (13), it will also be a solution of the Galerkin equations (21). This equivalence of Nyström's method and the discrete Galerkin method (see also [6]) together with our uniqueness assumption completes the proof.

Remarks. (1) For first-kind equations $(\lambda=0)$, the result holds even if the Gramian is not integrated exactly.

(2) Prenter [11] has shown that the full discretization of Nyström's method leads to the same linear system of equations as the collocation method suggested in her paper. This collocation method approximates the solution in a space of spline functions (piecewise Lagrange polynomials). The collocation points are the knots, and the quadrature formula integrates all functions in the approximating subspace exactly.

Clearly, the theorem applies to the classical Fourier method of Sections 1 and 2. Indeed, the trapezoidal rule integrates trigonometric polynomials of appropriate degree exactly. Another example to which the theorem can be applied is the following. Take the Legendre polynomials of degree $\leqslant N-1$ as the orthonormal Chebyshev system, and the $N$-point Gauss-Legendre quadrature formula for $Q$ (see, e.g., [1]). The Gramian is computed exactly, because polynomials of degree $\leqslant 2 N-$ 1 are integrated exactly.

Acknowledgments. Hermann Brunner, Walter Gautschi, and Martin Gutknecht provided valuable comments and references. Nick Trefethen is responsible for improvements in the presentation. Peter Henrici and his work were a source of inspiration. To them all, our warm thanks.

\footnotetext{
Department of Mathematics

University of California at San Diego

La Jolla, California 92093

Department of Mathematics

Massachusetts Institute of Technology

Cambridge, Massachusetts 02139
} 
1. M. Abramowitz \& I. A. Stegun, Handbook of Mathematical Functions, Dover, New York, 1965.

2. K. E. Atkinson, A Survey of Numerical Methods for the Solution of Fredholm Integral Equations of the Second Kind, SIAM, Philadelphia, Pa., 1976.

3. J.-P. BERRUT, Integralgleichungen und Fourier-Methoden zur numerischen konformen Abbildung, Ph. D. Thesis, ETH Zürich, 1985.

4. J.-P. BerRut, "A Fredholm integral equation of the second kind for conformal mapping," $J$. Comput. Appl. Math., v. 14, 1986, pp. 99-110.

5. J.-P. BERRUT, "Baryzentrische Formeln zur trigonometrischen Interpolation. I," J. Appl. Math. Phys. (ZAMP), v. 35, 1984, pp. 91-105.

6. L. M. Delves \& J. L. Mohamed, Computational Methods for Integral Equations, Cambridge University Press, Cambridge, 1985.

7. P. HENRICI, "Fast Fourier methods in computational complex analysis," SIAM Rev., v. 21, 1979, pp. $481-527$.

8. P. HeNRICI, Essentials of Numerical Analysis, Wiley, New York, 1982.

9. P. HenRICI, "Barycentric formulas for interpolating trigonometric polynomials and their conjugates," Numer. Math., v. 33, 1979, pp. 225-234.

10. N. Kerzman \& M. R. Trummer, "Numerical conformal mapping via the Szegö kernel," J. Comput. Appl. Math., v. 14, 1986, pp. 111-124.

11. P. M. Prenter, “A collocation method for the numerical solution of integral equations," SIAM J. Numer. Anal., v. 10, 1973, pp. 570-581.

12. L. REICHEL, "A fast method for solving certain integral equations of the first kind with application to conformal mapping," J. Comput. Appl. Math., v. 14, 1986, pp. 125-142.

13. M. SCHLEIFF, “Über Näherungsverfahren zur Lösung einer singulären linearen Integrodifferentialgleichung," Z. Angew. Math. Mech., v. 48, 1968, pp. 477-483.

14. I. Singer, Best Approximation in Normed Linear Spaces by Elements of Linear Subspaces, Grundlehren der mathematischen Wissenschaften, vol. 171, Springer, Berlin and New York, 1970.

15. G. T. SYMM, “An integral equation method in conformal mapping," Numer. Math., v. 9, 1966, pp. $250-258$.

16. M. R. Trummer, "An efficient implementation of a conformal mapping method based on the Szegö kernel,” SIAM J. Numer. Anal., v. 23, 1986, pp. 853-872. 